\title{
Statistical Finite Element Model for Bone Shape and Biomechanical Properties
}

\author{
Laura Belenguer Querol ${ }^{1}$, Philippe Büchler ${ }^{1}$, Daniel Rueckert ${ }^{2}$, \\ Lutz P. Nolte ${ }^{1}$, and Miguel Á. González Ballester ${ }^{1}$ \\ ${ }^{1}$ MEM Research Center, University of Bern, Switzerland \\ \{laura.belenguer, philippe.buechler, lutz.nolte, \\ miguel.gonzalez\} @memcenter.unibe.ch \\ ${ }^{2}$ Visual Information Processing, Department of Computing, \\ Imperial College London, UK \\ dr@doc.ic.ac.uk
}

\begin{abstract}
We present a framework for statistical finite element analysis combining shape and material properties, and allowing performing statistical statements of biomechanical performance across a given population. In this paper, we focus on the design of orthopaedic implants that fit a maximum percentage of the target population, both in terms of geometry and biomechanical stability. CT scans of the bone under consideration are registered non-rigidly to obtain correspondences in position and intensity between them. A statistical model of shape and intensity (bone density) is computed by means of principal component analysis. Afterwards, finite element analysis (FEA) is performed to analyse the biomechanical performance of the bones. Realistic forces are applied on the bones and the resulting displacement and bone stress distribution are calculated. The mechanical behaviour of different PCA bone instances is compared.
\end{abstract}

\section{Introduction}

Current orthopaedic implant design techniques make use of limited information about the target bone, such as a set of shape parameters (e.g. lengths and angles), which fail to capture the complexity of real anatomical shapes. Furthermore, evaluation and optimization of the implant is done by manual fitting and fixation procedures, applied on a small set of cadaver bones in a trial-and-error process to find the optimal shape and mechanic properties. Thus, it is difficult to assess whether the set of implant shapes will fit most of the population. The use of poorly fitting implants may lead to bone wear and the need for revision surgery to replace the implant. The availability of a statistical model of bone shape and biomechanical properties across a target population would allow to virtually test the implants on a representative set of bones, generated by sampling the statistical model. Thus, it would be possible to optimise implant shape as to fit a maximum of the target population.

This work proposes a methodology to perform statistical finite element analysis. We first build a statistical model combining bone shape and density information, via principal component analysis of CT images (in which Hounsfield units are 
proportional to bone density). This model will contain the average bone and the principal modes of variation, spanning a probability density function in a combined shape and intensity space, which allows generating further valid instances of bones. Afterwards, finite element analysis (FEA) is performed by simulating a set of forces acting on the bone, thus obtaining a statistical distribution of the stress (or other biomechanical response measures) at all points within the bone. This allows performing statistical statements of biomechanical performance across a given population.

Section 2 describes the methodology for construction of the combined statistical model of shape and intensity. Finite element analysis based on this model, and in particular its use for statistical assessment of bone stress under particular force loading conditions, are explained in Section 3. Results are reported in section 4. Finally, discussion and directions for future work are provided.

\section{Statistical Shape and Intensity Model}

Prior to model construction, the original image data (in our case CT images) are segmented to isolate the structure under consideration. Based on this segmentation, a mask is applied so only points within the region of interest are considered for further processing.

Correspondences are established between shapes by non-rigid registration. One of the images is taken to be the reference image, which will be deformed non-rigidly to the remaining instances. In order to rule out biases induced by the choice of the registration algorithm, we have tested two different registration methods, ITK [10] and Pasha [2], finding the results almost identical (Fig. 1).
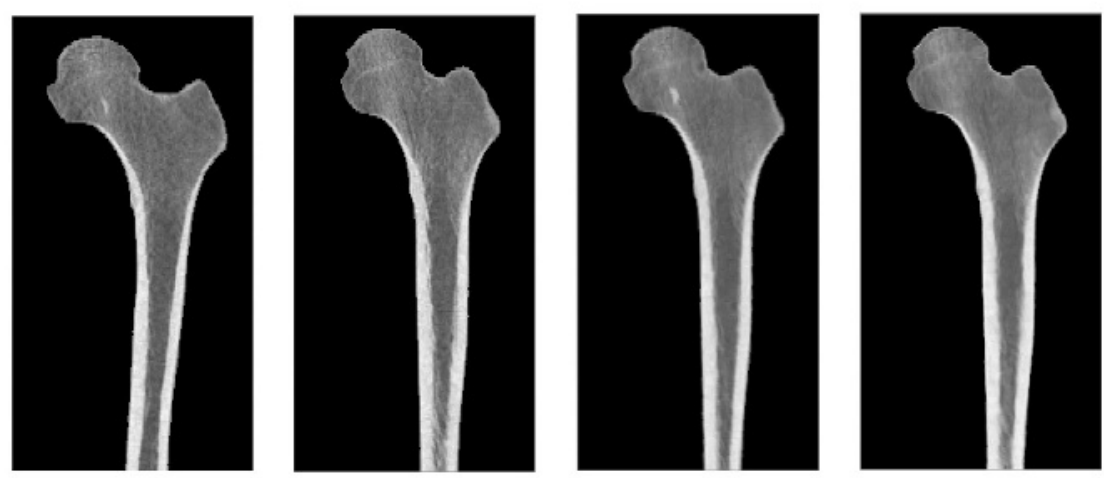

Fig. 1. Non-rigid registration examples using Pasha and ITK. From left to right: Reference and target masked images; Non-rigid registration with Pasha; Non-rigid registration with ITK.

In a second step, based on the deformation fields obtained from the registration process, we build vectors of corresponding positions and image intensities. The reference image can be described as in [8]: 


$$
c_{\text {REF }}=\left(x_{1}, y_{1}, z_{1}, I_{1}, \ldots, x_{n}, y_{n}, z_{n}, I_{n}\right)^{T}
$$

where $n$ is the number of voxels in the region of interest and $I_{i}$ is the intensity at voxel $\left(x_{i}, y_{i}, z_{i}\right)$. Similarly, each of the other images can be described as a vector of the same length:

$$
\begin{gathered}
c_{j}=\left(x_{1}+\Delta x_{1}^{j}, y_{1}+\Delta y_{1}^{j}, z_{1}+\Delta z_{1}^{j}, I_{1}^{j}, \ldots\right. \\
\left.x_{n}+\Delta x_{n}^{j}, y_{n}+\Delta y_{n}^{j}, z_{n}+\Delta z_{n}^{j}, I_{n}^{j}\right)^{T}
\end{gathered}
$$

where $\left(\Delta x_{i}^{j}, \Delta y_{i}^{j}, \Delta z_{i}^{j}\right)$ is the displacement vector at position $\left(x_{i}, y_{i}, z_{i}\right)$, and $I_{i}^{j}$ is the intensity of the voxel $\left(x_{i}+\Delta x_{i}^{j}, y_{i}+\Delta y_{i}^{j}, z_{i}+\Delta z_{i}^{j}\right)$ in image $j$.

The method used to compute a statistical description of the shape and intensity vectors is principal component analyis (PCA). We thus, obtain the average vector of the positions and intensities, $\bar{c}$, and the principal modes of variation, as the columns of the matrix $\phi$, computed by singular value decomposition of the covariance matrix. For compactness, the number of these vectors can be reduced by setting a threshold in the variance to be explained by our statistical model [4]. New instances of the model can be computed as linear combinations of the modes of variation:

$$
c=\bar{c}+\varphi b
$$

where $b$ is a vector of scalar coefficients modulating the contribution of each mode of variation. This effectively generates new images depicting valid bone shapes and corresponding bone densities.

\section{Finite Element Analysis}

Finite Element (FE) analysis is a numerical technique to solve partial differential equations over domains of complex shapes. FE techniques find a natural application in biomechanical studies, such as is the case for structural analysis of orthopaedic implants. FE models are useful to assess the design, position and fixation of new implants [1].

A technique to generate FE models representing the target population in terms of shape and mechanical properties is proposed in this study. The statistical models obtained in the former step, average and modes of variation are used to create 3D bone solids representing instances of the population. The obtained bone geometries are then meshed with a finite number (about 3500) of 3D tetrahedrons. Four FE nodes, one for each corner, define the shape of the elements.

The mechanical properties used in the model are inhomogeneous and depend on the bone density distribution. Since calibrated CT scans were used for the construction of the statistical model, instances of the model will maintain a proportional relationship between the bone relative density and the grey level in the 
images. It has been shown that the bone's Young's modulus can be obtained directly from the bone density $[3,9]$ :

$$
E= \begin{cases}60+900 \rho^{2}, & \rho<0.46\left(\mathrm{~g} / \mathrm{cm}^{3}\right) \\ 2875 \rho^{3}, & \text { otherwise }\end{cases}
$$

where $E$ is the Young's modulus in MPa and $\rho$ is the bone relative density $\left(\mathrm{g} / \mathrm{cm}^{3}\right)$.

The Poisson ratio is chosen equal to 0.3 , because this parameter is not dependent on bone density. The loading conditions correspond to a $1600 \mathrm{~N}$ force (2 times body weight) on the humeral head while the diaphyseal part of the bone is maintained fixed.

The displacement and bone stress distribution corresponding to the defined loading condition is calculated, and they lead to define bone stiffness, as the ratio between the force and the displacement, $k=F / d$. The average and variation of the stiffness over the population is obtained.

\section{Results}

The combined statistical model was computed from $11 \mathrm{CT}$ proximal femur data sets, which were cropped to only contain the region of the femur (ROI dimensions $175 \times 150 \times 200$, voxel size $\left.1 \times 1 \times 1.65 \mathrm{~mm}^{3}\right)$. The femur was segmented and other structures were masked as to not affect the registration. As mentioned in Section 2, one of the CT scans was used as the reference and the other images are registered to it. PCA is then applied to obtain the mean and modes of variation of combined shape and intensity. The top row of Fig.2 shows the mean and the effect of applying the first mode of variation ( \pm 2 standard deviations).

In a next step, we transfer the results obtained from the statistical model to create 3D bone solids, defined by a finite number of tetrahedrons (generated using Amira ${ }^{\circledR}$, Mercury Computing Systems, USA). To obtain the mechanical properties we read the intensity values at the mesh node coordinates. We obtain their relative density and the Young's modulus as described in (4). The middle row of Fig. 2, shows the relative bone density for the bones generated from the instances of the statistical model when applying the first mode of variation. It can be observed that the larger variations in shape and density are focused on the cortical area.

The loading setup defined in Section 3 is implemented and solved using Patran (MSC Software, USA). The von Misses stress for each of the bone instances generated above is shown in the bottom row of Fig. 2. These meshes represent the variation in biomechanical properties as consequence of the modulation of the first principal mode of variation of the combined statistical model.

The computed global stiffness for the three bone instances under vertical loading are (from left to right: $-2 \mathrm{sd}$, mean and $+2 \mathrm{sd}$ ): $0.36 \mathrm{~N} / \mathrm{mm}^{2}, 0.54 \mathrm{~N} / \mathrm{mm}^{2}, 2.14 \mathrm{~N} / \mathrm{mm}^{2}$. It can be observed that the variation induced by the first mode of variation leads to a change in the angle of the femoral shaft and reduced bone thickness. This affects the overall stability of the bone, as can be seen by the increase in the stress of the cortical bone (Fig.2, bottom), and the sharp increase in stiffness for the +2 sd case (right). 

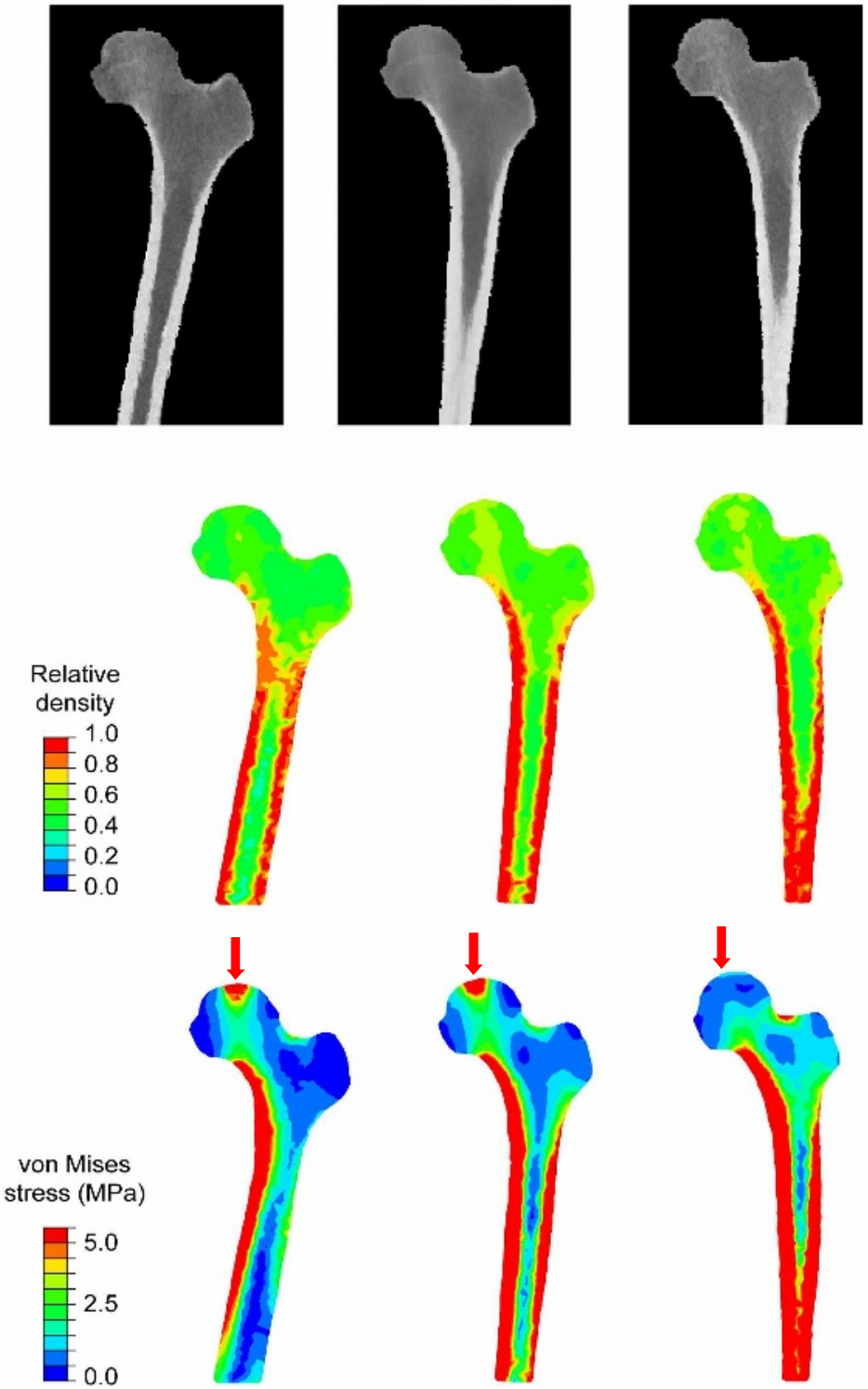

Fig. 2. Combined statistical model of shape and intensity and mechanical properties. From left to right: -2 sd, average and +2 sd. From top to bottom: combined statistical model of shape and intensity; relative density distribution; stress distribution corresponding to a vertical load of $1600 \mathrm{~N}$ applied at the arrow locations.

\section{Discussion and Conclusions}

We presented a framework for statistical biomechanical assessment including a combined statistical model of shape and intensity, in combination with finite element 
analysis. The application of our methods for modelling bone shape and mechanical behaviour has been shown.

The statistical model construction is based on non-rigid registration. This has the advantage that no landmarks or parametric representations need to be defined. By combining shape and intensity information into the same PCA evaluation, the model contains information about the correlation between shape and density. This could allow to predict bone density when only shape is known, and this is one of our directions of future work.

The FE analysis shown in this work could be improved by addressing a set of experiment setup issues. First, we employed tetrahedral meshes, which are known to have poor accuracy as compared to other element types. Further, the bone geometries were meshed independently from each other, resulting in different FE meshes for the different bones. This leads to difficulties in the direct comparison of the stress distribution between the different bone shapes under consideration, as well as discrepancies in the location of the loading forces applied. The alternative is to deform a pre-defined mesh, such as is done by [12]. However, it is extremely difficult to ensure the validity of the mesh for further FEA under arbitrary deformations.

The target application is orthopaedic implant design. Virtual testing of new implants will in the future replace cadaver testing. Further, being able to study the whole range of bone shapes and densities of the target population will lead to better fitting implants, as well as a considerable cost reduction in the design process. In order to assess the appropriateness of an implant, further development should be done to define the different scenarios of the implant, in terms of positions were the it is likely to be placed and the force loading conditions.

A complementary application of these techniques will be the patient-specific preclinical evaluation of an implant, taking into account the particular skeletal anatomy, bone quality, and implant position to assess the biomechanical performance of the implant on the patient.

\section{Acknowledgements}

We would like to thank Dr. Xavier Pennec from INRIA for sharing his non-rigid registration software, Pasha.

This research was funded by the Swiss National Science Foundation through its National Center of Competence in Research (NCCR) on Computer Aided and Image Guided Medical Interventions (http://co-me.ch).

\section{References}

1. Büchler, P., and Farron, A., Benefits of an Anatomical Reconstruction of the Humeral Head During Shoulder Arthroplasty: A Finite Element Analysis. Clinical Biomechanics, 19 (1):16-23, 2004

2. Cachier, P., Bardinet, E., Dormont, D., Pennec, X., and Ayache, N.: Iconic Feature Based Nonrigid Registration: The PASHA Algorithm. Computer Vision and Image Understanding. Special Issue on Nonrigid Registration, 89(2-3):272-298, February.March, 2003. 
3. Carter, D.R., and Hayes, W.C.: The compressive behavior of bone as a two-phased porous structure, Journal of Bone and Joint Surgery, 58A(7), pp. 954-962, 1977.

4. Cootes, T.F. and Taylor C.J.: Statistical models of appearance for medical image analysis and computer vision. Proc. SPIE Medical Imaging, 2001.

5. Cootes, T.F., Edwards, G.J. and Taylor, C.J.: Active Appearance Models. IEEE Transactions on Pattern Analysis and Machine Intelligence, Vol.23, No.6, pp.681-685, 2001.

6. Cootes, T.F., Taylor C.J, Cooper, D.H and Graham, J.: Active shape models - Their training and application, Computer Vision Image Understanding, vol 61, no 1, pp 38-59, 1995.

7. Frangi, A.F., Rueckert, D., Schnabel, J.A., and Niessen, W.J.: Automatic construction of multiple-object three-dimensional statistical shape models: Application to cardiac modeling. IEEE Transactions on Medical Imaging, 21(9):1151-1166, 2002.

8. González Ballester, M.A., Pennec, X., Linguraru, M.G. and Ayache, N.: Generalised image models and their application as statistical models of images. Medical Image Analysis, 8(3) 361-9, 2004.

9. Rice, J.C., Cowin, S.C. and Bowman, J.A.: On the dependence of the elasticity and strength of cancelous bone on apparent density, Journal of Biomechanics, Vol. 21(2), pp. 155-168, 1988.

10. Rueckert, D., Frangi, A.F., and Schnabel, J.A.: Automatic construction of 3D statistical deformation models of the brain using non-rigid registration. IEEE Transactions on Medical Imaging, 22(8):1014-1025, 2003.

11. Rueckert, D., Sonoda, L.I., C. Hayes,. Hill, D.L.G., Leach, M.O., and Hawkes, D.J.: Nonrigid registration using free-form deformations: Application to breast MR images. IEEE Transactions on Medical Imaging, 18(8):712-721, 1999.

12. Yao, J. and Taylor,R.: Tetrahedral Mesh Modeling of Density Data for Anatomical Atlases and Intensity-Based Registration. Proc. MICCAI 2000. Pittsburgh, PA, USA: Springer, 2000 . 